\title{
The utility of a local multidisciplinary working group to oversee the establishment of rapidly evolving standards of care and to support trial recruitment during the COVID-19 pandemic
}

\author{
Authors: Giovanni Satta, ${ }^{\mathrm{A}}$ Taryn Youngstein, ${ }^{\mathrm{B}}$ Liz Lightstone ${ }^{\mathrm{C}}$ and Mark Gilchrist ${ }^{\mathrm{D}}$ on behalf of the COVID-19 \\ treatment guidelines working group at Imperial College Healthcare NHS Trust
}

Coronavirus disease 2019 (COVID-19) was first identified in December 2019 in Wuhan, China. The first analyses of cases described high numbers of critically ill patients requiring intensive care admission with significant late inflammatory features. By the time the first cases of SARS-CoV-2 infection were diagnosed in the UK, a wide range of drugs were under consideration and it became clear that the input of clinicians covering all organ systems (in particular, infectious diseases, haematology, rheumatology, renal medicine and intensive care) and of expert specialist pharmacists was necessary at the local level. Thus, an expert multidisciplinary (MDT) group within our organisation was convened to offer a standardised approach and robust clinical governance for the treatment of COVID-19 patients admitted to our hospitals and rapidly develop standards of care as evidence evolved. This commentary explores the methods and mechanisms for creating an MDT COVID-19 treatment working group which are applicable to any hospital likely to admit and care for high numbers of COVID-19 patients and demonstrates how the structure and governance of the group allowed for rapid adoption of both dexamethasone and tocilizumab into standard of care as data became available.

KEYWORDS: COVID-19, SARS-CoV-2, multi-disciplinary

DOI: $10.7861 /$ clinmed.2020-1021

\section{Introduction}

Coronavirus disease 2019 (COVID-19) was first identified in December 2019 in Wuhan, China. The first analyses of cases

Authors: Aconsultant in infection, Imperial College Healthcare NHS Trust, London, UK; ${ }^{\mathrm{B}}$ Consultant rheumatologist, Imperial College Healthcare NHS Trust, London, UK; ' professor of renal medicine, Imperial College Healthcare NHS Trust Centre for Inflammatory Disease, London, UK and Imperial College London, London, UK; ${ }^{D}$ consultant pharmacist in infectious disease, Imperial College Healthcare NHS Trust, London, UK described high numbers of critically ill patients requiring intensive care admission with significant late inflammatory features. ${ }^{1}$ No licenced treatments were available and a multitude of experimental and repurposed drugs rapidly came under investigation. Within the UK, hospitals were directed by the chief medical officer (CMO) to enrol all eligible patients into fast-tracked COVID-19 clinical trials. ${ }^{2}$ This approach has allowed rapid assimilation of a large evidence base and has been world-leading, in particular considering the RECOVERY and REMAP-CAP trial results. ${ }^{3,4}$

The first emerging therapies from Europe and Asia were those repurposed from other indications; the anti-malarial and anti-rheumatic hydroxychloroquine, the anti-retroviral lopinavirritonavir, and the targeted cytokine inhibitors tocilizumab (interleukin- 6 receptor antagonist) and anakinra (interleukin-1 receptor antagonist), both of which are licensed for treatment of rheumatological and haematological disease. By the time the first cases of SARS-CoV-2 infection were diagnosed in the UK, a wide range of drugs were under consideration and it became clear that the input of clinicians covering all organ systems (in particular, infectious diseases, haematology, rheumatology, renal and intensive care) and expert specialist pharmacists was necessary at local level. Thus, an expert multidisciplinary (MDT) group within our organisation was convened to establish a standardised approach and robust clinical governance for the treatment of COVID-19 patients admitted to our hospitals and to rapidly develop standards of care as evidence evolved.

This commentary explores the process for creating an MDT COVID-19 treatment working group and demonstrates how the structure and governance of the group allowed rapid adoption of both dexamethasone and tocilizumab into standard of care as data became available. We describe the challenges of implementing rapidly evolving guidance, including the treatment of challenging cases ineligible for clinical trials. Our experiences are applicable to any hospital likely to admit and care for high numbers of COVID-19 patients.

\section{Setting up a multidisciplinary working group}

Our organisation is a large academic healthcare organisation in London providing acute secondary and tertiary care across three large hospital sites. It serves a population of over 2 million 
people and has more than 1,000 inpatient beds covering all major medical, surgical adult and paediatric specialities. The group was initially formed from within the infection and respiratory teams admitting the first COVID-19 cases. Its remit was to provide guidance on the clinical management of patients infected with SARS-CoV-2 to other medical and pharmacy teams caring for these patients, due to the high level of clinical queries resulting from the rapidly evolving nature of the pandemic. As the pandemic developed, it soon became evident that the type of questions arising required expertise from a broader team, and so an expanded multidisciplinary working group was formed, including stakeholders from rheumatology, haematology, critical care, renal medicine, paediatrics and obstetrics to reflect the complexity of the cases being managed. It was also decided to appoint two leads to co-chair this new group, a physician and a consultant pharmacist, based on an approach previously adopted within antimicrobial stewardship structures. There is already evidence to support a multidisciplinary approach for the treatment of other patient groups with complex conditions such as cancer and the elderly, 5 but evidence for COVID-19 is also emerging. ${ }^{7.8}$

By definition, a 'working group' is a group of experts working together to achieve specified goals. Thus, the group defined a mission statement with official terms of reference and clear objectives, which were to provide guidance to frontline clinicians and pharmacists, scrutinise potential new treatments and develop an evidence-based approach to their use, and provide expert opinion on the use of off-label and experimental treatments. The group worked in parallel with a newly established COVID clinical trials group and both of these groups reported to the hospital's core pandemic management committee. The medical director's office maintained overall control of approval of any change to the treatment guidelines and ensured that guidance was always prioritised and that all patients were assessed for eligibility for clinical trial entry. Once an approach to treatment was approved, the medical director's office was happy to devolve the daily clinical decisions back to the group, on the condition of presenting a weekly summary and discussion of patient treatment in conjunction with available drugs supplies. This latter issue was particularly important, as one major concern was to maintain drug supplies for patients already under our care taking these medications for approved indications for their chronic disease.

Key practical considerations when setting up an MDT working group are set out in Box 1.

\section{Outputs of the multidisciplinary working group}

The main output of the group was the creation of clear treatment guidelines for the entire organisation. The guidelines had to be practical, covering essential basics on how to manage COVIDpositive patients, from oxygen and anticoagulation to antibiotic stewardship (aspects of care covered are given in Box 2). The guidelines became a local standard of care (end of March 2020) and have been continuously adapted since then in response to changing evidence (version 10 at time of submission). Finally, the guidelines covered the experimental treatments available, with a rationale for their utilisation and the clinical trials offering them.

An additional role of the group was to implement and provide governance on off-label and compassionate use of trial medication in patients ineligible for clinical trials. As a regional centre for nephrology, with services including nephritis, dialysis and renal transplantation, as well as services for complex haematology
Box 1. Checklist for setting up a new multidisciplinary COVID-19 treatment working group

\section{Engage stakeholders}

$>$ Identify and engage all relevant specialties (for our working group, these included infectious diseases, microbiology and virology, specialist clinical pharmacy, respiratory medicine, rheumatology, haematology, renal medicine, intensive care, paediatrics and obstetrics)

Agree terms of reference

$>$ Clearly define the roles and objectives of the group, write a mission statement and set out your expected outputs

$>$ Define your membership and quorum

$>$ Establish a frequency for meetings

\section{Establish governance and accountability}

$>$ Obtain the medical director's approval and endorsement

$>$ Ensure there is a clear clinical governance structure and a defined position in the organisational flowchart

$>$ Establish reporting responsibilities (in our case this was directly to the medical director's office)

> Set up processes for documentation, including a clear record of all referrals received and of clinical advice given by the group

Ensure effective leadership and values

> Flatten hierarchies to encourage open and equal debate among all members of the group

$>$ Co-chairing of the group can be beneficial to provide a balanced view

$>$ Set a clear set of values to be followed during meeting (eg kind, expert, collaborative and aspirational)

Ensure visibility and accessibility to other clinical teams

> Make sure guidelines are easily accessible on the hospital intranet

$>$ Provide easy-to-use flowcharts

> Set up a simple referral system (form to be emailed or online referral)

> Establish a 24/7 on-call rota for a responsive service (including essential pharmacy support to release drugs in stock within hours of original referral)

Secure drug supplies

> Establish collaborative partnerships with pharmaceutical companies for compassionate supply of drugs

> Make sure all compassionate use request forms are completed and sent back to pharmaceutical companies

$>$ Carry out a daily check of pharmaceutical stock to replenish drug supplies

Box 2. Aspects of care covered by guidelines released

by the COVID-19 treatment guidelines working group at Imperial College Healthcare NHS Trust

$>$ The recommended set of laboratory tests to be performed

$>$ Infection control precautions

$>$ Antimicrobial stewardship in the context of COVID 19

$>$ Routine ward round checklist

$>$ Ceiling of care and treatment expectations

$>$ When to refer to intensive care

$>$ Anticoagulation

$>$ Clinical trials available

> Compassionate use when trials are not available 
and stem cell transplantation and complex rheumatology care, including inflammatory multisystem disease, a large number of our own patients were not eligible for the immunomodulatory arms of our open clinical trials. Using local expertise and only when patients were ineligible for clinical trials, the group oversaw the prescription of off-label or compassionate use of steroids, tocilizumab, anakinra and ruxolitinib in those cases who were critically unwell with COVID-19. The outcomes of these patients have already been reported or are currently under review., ${ }^{9,10}$ The group also oversaw the introduction of the Early Access Scheme for the antiviral remdesivir and reviewed all requests within the hospital for compassionate use of this medication in pregnancy and paediatric patients. ${ }^{11}$

To date there have been around 200 referrals of clinical cases to our group with universal positive feedback from clinicians caring for these complex patients in all settings.

\section{Case study 1: Dexamethasone}

In the early phase of the disease, there was uncertainty regarding the efficacy of steroid use in the treatment of COVID-19. With emerging evidence ${ }^{3,4}$ and a national therapeutic alert, ${ }^{12}$ the group rapidly deployed central messaging around steroid guidance and stock mobilisation across all admitting areas within 24 hours. Additional guidance regarding side effects and their management was issued. In the second wave of the pandemic, the group monitored emerging evidence on the utility of higher-dose steroid use (including any further national alerts) and again were able to collaborate rapidly and discuss with colleagues to ensure appropriate use.

\section{Case study 2: Tocilizumab}

Early reports suggested that tocilizumab might be of benefit in COVID-19.13 The group continuously updated its guidance on the basis of best available evidence, including national alerts. All referrals for tocilizumab were directed through the group to ensure appropriateness and to maintain supply/protect stock for existing chronic patients. The group operated $8 \mathrm{am}$ to $11 \mathrm{pm}, 7$ days a week, with a weekly rota including specialist pharmacists and doctors. Decisions were documented in the clinical notes and emailed to the referrer. In the 20 days from NHS England updating national advice around IL6 inhibitors the group received approximately 100 referrals, of which $80 \%$ were approved.

\section{Conclusions}

The results of hundreds of clinical trials for COVID-19 are emerging and, as approval comes through centrally, there is a requirement for local scrutiny over the safe and rapid implementation and governance of these medications. Despite the existence of many other clinical groups, they are not generally sufficiently multidisciplinary or agile to be fit for purpose in the setting of a novel pandemic infection. COVID-19 may also lie outside their mission statements and terms of reference. The presence of a COVID-19 treatment guidelines working group can provide some reassurance on the appropriate use of drugs and a standardised approach to treatment.

Based on the above, we recommend the creation of a local MDT COVID-19 treatment working group in each hospital admitting COVID patients. Some smaller hospitals (district general hospitals or highly specialised units) may not have all the necessary specialties and expertise on site and will need to create a network with their referral centres (in our case, we also provided clinical opinions for some external referrals).

\section{Acknowledgements}

The authors would like to acknowledge the contributions of the other members of the multidisciplinary working group to the work described in this paper: Dr Frances Akor, Mr Ahmad Al-Abdulla, Professor Jane Apperley, Professor Stephen Brett, Dr Aristeidis Chaidos, Mrs Aneeka Chavda, Professor Graham Cooke, Dr Nichola Cooper, Amy Frances, Professor Anthony Gordon, Professor Anastasios Karadimitris, Professor Prapa Kanagaratnam, Ms Priti Kukadia, Professor Mike Laffan, Mr Christoph Lees, Dr Roseanne Meacher, Dr Dragana Milojkovic, Dr David Muir, Mr Mikin Patel, Nickisha Patel, Mr Charles Parkinson, Dr Clare Ross, Dr Francesca Rubulotta, Dr Elizabeth Whittaker and Dr Michelle Willicombe. The authors would also like to thank the Executive Team at Imperial College Healthcare NHS Trust for their support during the COVID-19 pandemic and all our clinical colleagues for referring patients.

\section{References}

1 Zhou F, Yu T, Du R et al. Clinical course and risk factors for mortality of adult inpatients with COVID-19 in Wuhan, China: a retrospective cohort study. Lancet 2020;395:1054-62.

2 National Institute of Health Research. UK's top doctors urge recruitment of COVID-19 patients for vital scientific research. NIHR, 2020. www.nihr.ac.uk/news/uks-top-doctors-urge-recruitment-ofcovid-19-patients-for-vital-scientific-research/24591.

3 RECOVERY Collaborative Group. Dexamethasone in hospitalized patients with Covid-19. N Engl J Med 2021;384:693-704.

4 REMAP-CAP Investigators. Interleukin- 6 receptor antagonists in critically ill patients with Covid-19. N Engl J Med 2021, in press (DOI:10.1056/NEJMoa2100433).

5 Abdulrahman GO, Jr. The effect of multidisciplinary team care on cancer management. Pan Afr Med J 2011;9:20.

6 To TH, Davies OJ, Sincock J, Whitehead C. Multidisciplinary care needs in an Australian tertiary teaching hospital. Aust Health Rev 2010;34:234-8.

7 Romei C, De Liperi A, Bartholmai B]. COVID-19: the importance of multidisciplinary approach. Acad Radiol 2020;27:1327-8.

8 Galluccio F, Ergonenc T, Garcia Martos A et al. Treatment algorithm for COVID-19: a multidisciplinary point of view. Clin Rheumatol 2020:39:2077-84.

9 Innes AJ, Cook LB, Marks S et al. Ruxolitinib for tocilizumabrefractory severe COVID-19 infection. Br J Haematol 2020, in press (doi: 10.1111/bjh.16979).

10 Chaidos A, Katsarou A, Mustafa C, Milojkovic D, Karadimitris A. Interleukin 6-blockade treatment for severe COVID-19 in two patients with multiple myeloma. Br J Haematol 2020;190:e9-e11.

11 Grein J, Ohmagari N, Shin D et al. Compassionate use of remdesivir for patients with severe Covid-19. N Engl J Med 2020;382:2327-36.

12 Medicines and Healthcare products Reporting Agency. Corticosteroids in the treatment of suspected or confirmed COVID19. MHRA, 2021. www.cas.mhra.gov.uk/ViewandAcknowledgment/ ViewAlert.aspx?AlertID $=103092$.

13 Alzghari SK, Acuna VS. Supportive treatment with tocilizumab for COVID-19: a systematic review. J Clin Virol 2020;127:104380.

Address for correspondence: Dr Giovanni Satta, Head of Specialty for Infection - Consultant in Infectious Diseases and Medical Microbiology, Imperial College Healthcare NHS Trust, St Mary's Hospital, W2 1NY, London, UK.

Email: Giovanni.satta@nhs.net 